\title{
DIVERSIDAD VEGETAL DE LAGUNAS TEMPORALES EN LA ESTEPA PATAGÓNICA CHILENA NOROCCIDENTAL
}

\author{
PLANT DIVERSITY OF TEMPORARY PONDS IN THE \\ NORTHWESTERN CHILEAN PATAGONIAN STEPPE
}

\section{Cristina San Martín ${ }^{1}$, Yessica Pérez, Miguel Alvarez², Christian Salazar 3 \& Carlos Ramírez ${ }^{4}$}

Las lagunas temporales son hábitats que fluctúan estacionalmente entre condiciones de anegamiento y sequía (Deil, 2005; Álvarez, 2008), ambas adversas para el crecimiento de las especies vegetales. Debido a ello, las plantas capaces de crecer en estos humedales presentan estrategias especializadas como lo son el enanismo y la plasticidad fenotípica (Álvarez, 2008). En Chile el conocimiento sobre la ecología y distribución geográfica de lagunas temporales es muy escaso y se remite a algunos estudios florísticos y vegetacionales (Bliss et al. 1998; Álvarez, 2008; San Martín \& Álvarez, 2009), mientras que para otros tipos de biocenosis (por ejemplo, zoocenosis) es prácticamente ausente. Estudios florísticos más detallados se han hecho en lagunas temporales del bioclima mediterráneo (sensu Luebert \& Pliscoff, 2006) y en la parte norte del bioclima templado (Bliss et al. 1998; Deil et al. 2007; Álvarez et al. 2012), pero se desconocen estudios hechos al sur del paralelo $44^{\circ} \mathrm{S}$. En este trabajo presentamos un catastro de especies de charcos temporales y lagunas semipermanentes (con nivel de agua fluctuante) colectadas durante el verano de 2011 al noreste de Coyhaique (Fig. 1).

En el lugar de estudio, el paisaje de estepa es característicamente ondulado y las depresiones son ocupadas por charcos temporales o semipermanentes, dependiendo de su tamaño. Este patrón ha sido mencionado para la Patagonia continental argentina, donde también se incluyen como típicos ambientes de lagunas temporales los cañadones (surcos de drenaje) y las cubetas de deflación (Thermokarst en alemán) (Roig et al. 1985a; Collantes \& Faggi, 1999; Martínez Carretero, 2004). La dinámica de inundación de estos humedales está determinada por la estacionalidad en las lluvias y el derretimiento de las nieves (Deil et al. 2011).

El paisaje en el que están insertas estas lagunas corresponde a planicies de depósitos fluvioglaciales de composición areno-limosa, los cuales se depositaron durante el Cuaternario (Pleistoceno y Holoceno), además resaltan los domos volcánicos establecidos durante el Cretácico. Estos domos son más frecuentes y caracterizan por ejemplo al paisaje del Valle de la Luna (Fig. 2A) (Servicio Nacional de Geología y Minería 2003¹, Gobierno Regional de Aysén y Gesellschaft für Technische Zuzammenarbeit 2005²).

\footnotetext{
${ }^{1}$ Servicio Nacional de Geología y Minería. (2003). Mapa Geológico de Chile.

2 Gobierno Regional de Aysén \& Gesellschaft für Technische
} Zuzammenarbeit. (2005). Atlas Región de Aysén.

\footnotetext{
${ }^{1}$ Universidad Austral, Instituto de Ciencias de la Tierra y Evolución, Valdivia, Chile.

${ }^{2}$ Rheinische Friedrich-Wilhelms-Universität, INRES-Vegetationsökologie, Bonn, Alemania.

${ }^{3}$ Museo Nacional de Historia Natural, Santiago, Chile.

${ }^{4}$ Pontificia Universidad Católica, Departamento de Ecología, Santiago, Chile. csanmart@uach.cl 


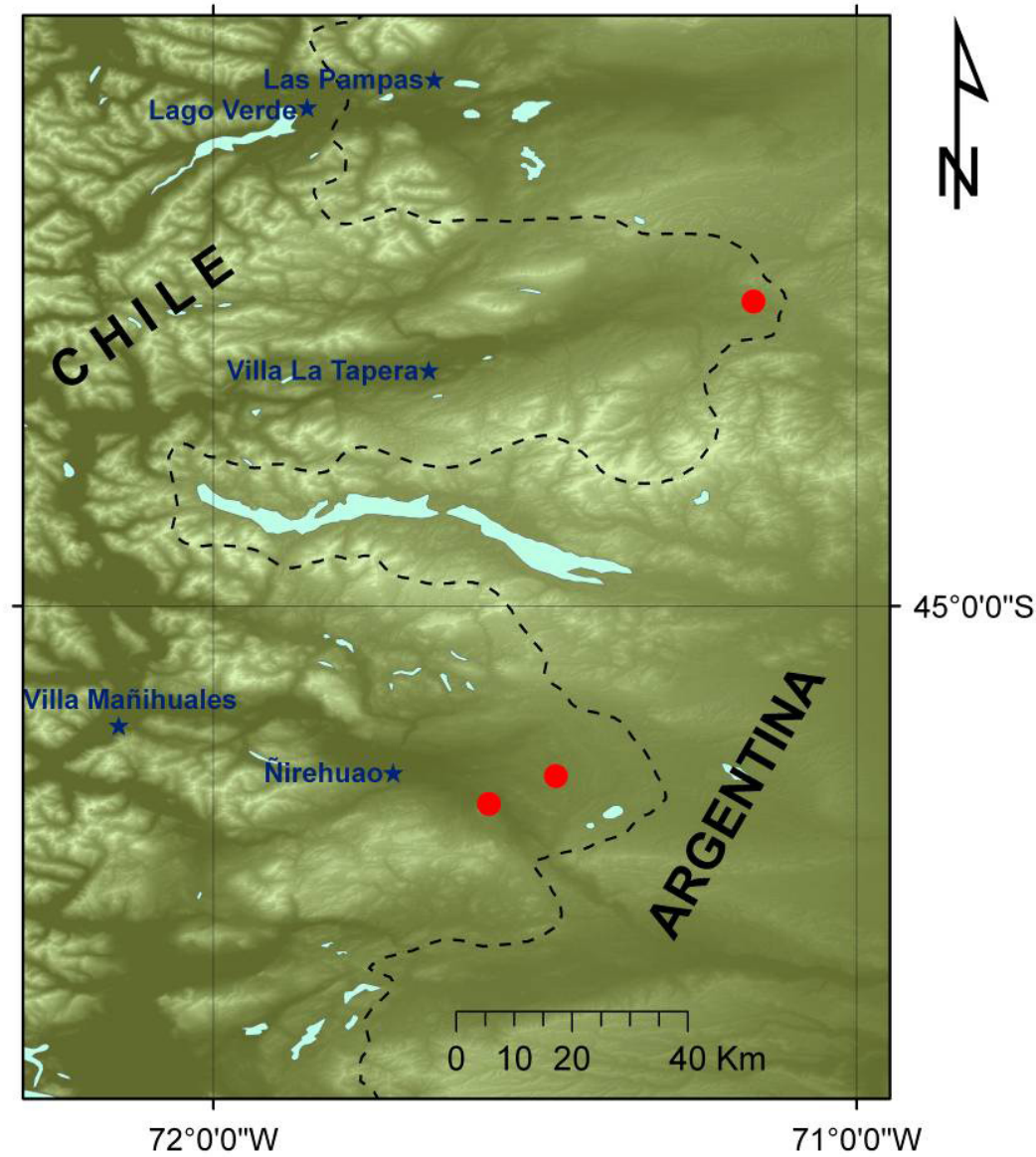

Fig. 1. Ubicación geográfica de los humedales temporales (puntos rojos) estudiados en la Patagonia chilena noroccidental. Línea discontinua representa el límite entre Chile y Argentina.

La vegetación típica de las planicies es la estepa magallánica o coironal (Roig, 1998), domida por Festuca gracillima, Rumex acetosella, Taraxacum officinale, Achillea millefolium y Acaena pinnatifida, esta comunidad ha sido clasificada dentro de la clase Festucetea gracillimae descrita por Roig et al. (1985b) y también es denominada estepa graminosa húmeda del distrito magallánico por León et al. (1998). La presencia de una gran cantidad de malezas alóctonas podría indicar un origen secundario producto de la degradación por pastoreo de un primitivo matorral de ñirre (Nothofagus antarctica). En depresiones húmedas se presentan los pastizales de la asociación Deschampsio-Hordeetum pubiflori (Méndez \& Ambrosetti, 1985; Roig et al. 1985b), dominados por Hordeum pubiflorum y Deschampsia caespi- tosa. En las depresiones aún más profundas y con inundación permanentemente, observamos una vegetación riparia dominada por Hippuris vulgaris (que es una especie de origen Europeo), Myriophyllum quitense, Schoenoplectus californicus, Juncus microcephalus, Potamogeton linguatus y Chara vulgaris. Esta a su vez ha sido denominada por Roig et al. (1985b) como Hippuretum vulgaris (clase Hippuretea vulgaris) y también corresponde a las comunidades de Hippuris-Myriophyllum según Clausen et al. (2006). En este mismo ambiente se presentan las comunidades de JuncusGlyceria, dominada por Juncus scheuchzerioides y Glyceria multiflora y acompañadas por H. vulgaris, $M$. quitense, $P$. linguatus y diversas especies del género Carex (Clausen et al. 2006).

Seis de las especies vegetales observadas 
Tabla 1. Especies vegetales observadas en lagunas temporales de la Patagonia Noreste. Origen (Or): introducida (i), nativa (n) y endémica (e). Formas de vida (FV): anual o bienal (T), perenne hemicriptófita (H). Distribución geográfica (Geogr) en los censos colectados por (Deil et al. 2011): Argentina (AR), Bolivia (BO), Chile (CL), Colombia (CO) y Perú (PE). Nomenclatura según Zuloaga et al. (2008).

\begin{tabular}{lllll}
\hline Especie & Familia & Or & FV Geogr \\
\hline Arenaria pleurantha Phil. & Caryophyllaceae & e & H & \\
Gnaphalium phaeolepis Phil. & Asteraceae & e & H CL \\
Hordeum pubiflorum Hook.f. & Poaceae & e & H AR \\
Lasthenia kunthii (Less.) Hook. et Arn. & Asteraceae & e & T CL \\
Limosella australis R.Br. & Plantaginaceae & n & T AR,BO,CL,CO,PE \\
Lilaeopsis macloviana (Gand.) A.W.Hill & Apiaceae & n & H AR,BO,CL,PE \\
Lobelia oligophylla (Wedd.) Lammers & Campanulaceae & n & H AR \\
Plagiobothrys calandrinioides (Phil.) I.M.Johnst. & Boraginaceae & e & T AR \\
Polygonum persicaria L. & Polygonaceae & i & H CL \\
Potentilla anserina L. & Rosaceae & i & H AR,CL \\
Ranunculus biternatus Sm. & Ranunculaceae & e & T AR,CL \\
Rorippa nana (Schltdl.) J.F.Macbr. & Brassicaceae & n & H AR \\
Rumex maritimus L. & Polygonaceae & i & T AR \\
Veronica peregrina L. subsp. xalapensis (Kunth) Pennell & Plantaginaceae & i & T AR,CL \\
\hline
\end{tabular}

en la vegetación efímera de las lagunas temporales estudiadas son endémicas (Tabla 1), vale decir, con una distribución geográfica restringida. El caso contrario lo representan las especies introducidas, que actualmente tienen una distribución cosmopolita. La presencia de ambos contingentes florísticos fitogeográficos es típico de lagunas temporales, como lo menciona Deil (2005). Considerando la presencia de las especies en una base de datos de censos vegetales en lagunas temporales de Sudamérica (Deil et al. 2011; Deil \& Alvarez, 2012), Limosella australis y Lilaeopsis macloviana son las que muestran una mayor amplitud en su distribución geográfica y que, a su vez, también han sido mencionadas como especies diagnósticas de la clase Limoselletea australis y de la alianza $\mathrm{Li}$ laeopsidion andinae (Lilaeopsis andina es sinónimo de L. macloviana, (Fig. 2B), respectivamente (Deil et al. 2011). Por supuesto, aquí sólo se consideran registros vegetacionales, pero también se puede obtener información complementaria si se suman los registros florísticos. Tal es el caso de Plagiobothrys calandrinioides (Fig. 2C), especie que es característica de humedales temporales en la Patagonia oriental argentina (Faggi, 1985; Roig et al. 1985a; Deil et al. 2011), pero que en Chile hasta ahora solamente había sido mencionada para las regiones Metropolitana y de Valparaíso (Horn 2000; Arroyo et al., 2002; Zuloaga et al., 2008). Más drástico es el cambio considerando a Rorippa nana (Fig. 2D), la cual también crece en el páramo colombiano (Parra \& Fernández, 2002), en el altiplano peruano-boliviano-argentino (Ruthsatz, 1977; Cooper et al. 2010; Fontúrbel et al. 2006, Ortuño et al. 2006) y en las islas Galápagos (Jaramillo Díaz et al. 2011). Puesto que esta especie no está incluída para Chile ni en la base de datos de Deil \& Alvarez (2012), ni en los correspondientes listados florísticos (Marticorena \& Quezada, 1985; Arroyo et al. 1990; Zuloaga et al. 2008), en este trabajo se hace su primera mención para la flora de Chile (Tabla 2). Por ende, esta especie muestra el mismo patrón de distribución a lo largo de la Cordillera de Los Andes, seguido por Limosella australis y Lilaeopsis macloviana.

De acuerdo a Deil et al. (2011), la vegetación de charcos temporales guarda estrecha relación con la alianza Plagiobothryo calandrinioidisAcaenion platyacanthae, la cual tiene un carácter pionero (Faggi, 1985). Por otro lado, las comunidades observadas al borde de lagunas semipermanentes están más asociadas a la alianza Pration repentis (Méndez \& Ambrosetti, 1985). Su nombre es derivado de Pratia repens (sinónimo de Lobelia 


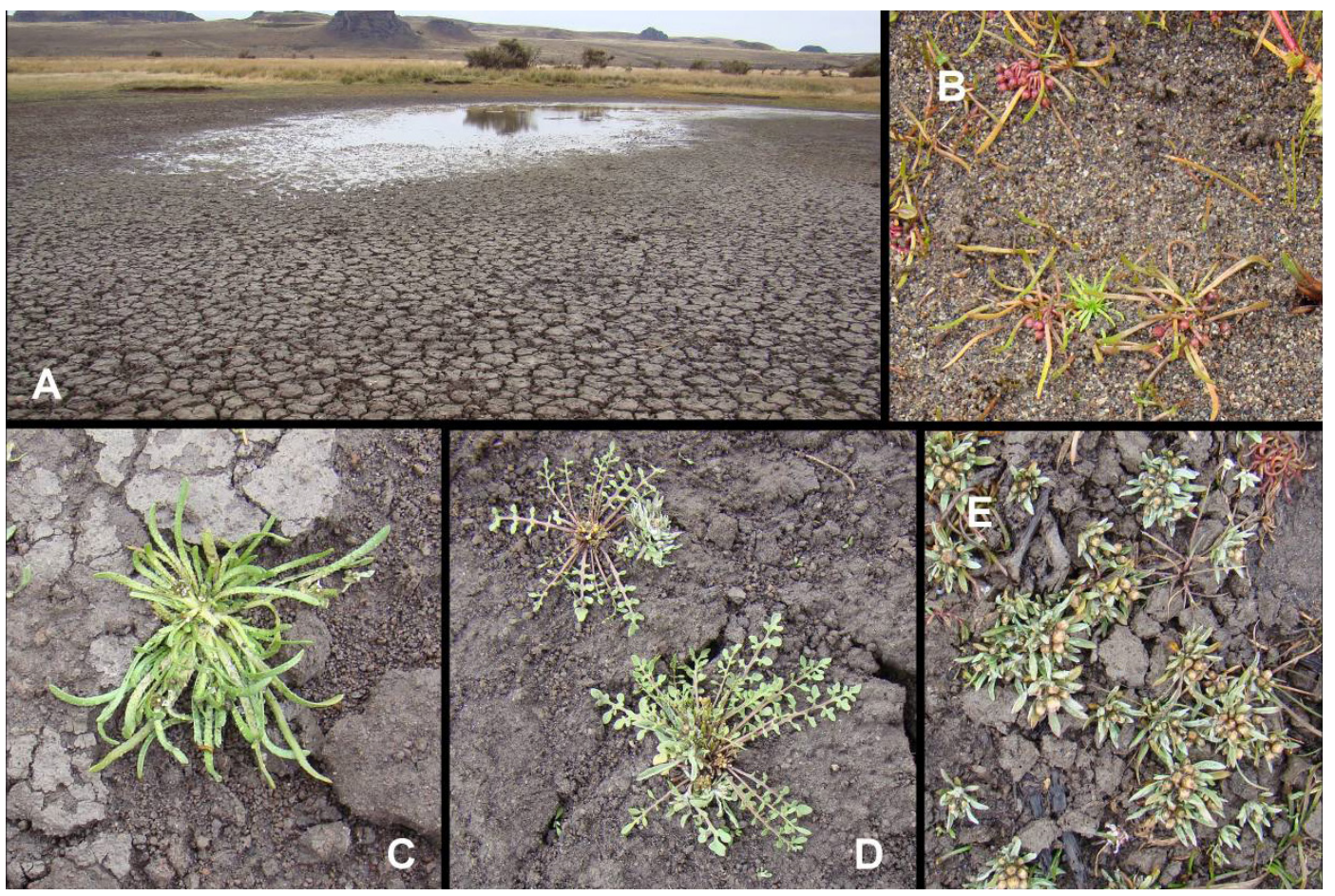

Fig. 2. Lagunas temporales de la Patagonia chilena Norte. A) Laguna en el Valle de la Luna, B) Lilaeopsis macloviana, C) Pla-giobothrys calandrinioides, D) Rorippa nana, E) Gnaphalium phaeolepis.

Tabla 2. Descripción de Rorippa nana (modificado de Parra \& Fernández, 2002).

\begin{tabular}{|c|c|c|c|}
\hline Especie & Descripción & Distribución & Material Colectado \\
\hline $\begin{array}{l}\text { Rorippa nana } \\
\text { (Schltdl.) J.F. } \\
\text { Macbr. }\end{array}$ & $\begin{array}{l}\text { Hierba pequeña, generalmente } \\
\text { en roseta (prostrada), solitarias o } \\
\text { agrupadas en cojines pequeños. } \\
\text { Aurículas de la base del pecíolo } \\
\text { (cuando presentes) } 0,6-0,7 \mathrm{~mm} \\
\text { de largo, } 0,3-0,4 \text { mm de ancho. } \\
\text { Hojas imparipinnadas } 2-4 \mathrm{~cm} \\
\text { de largo, } 0,7-1,2 \mathrm{~cm} \text { de ancho. } \\
\text { Folíolos laterales con un lóbulo } \\
\text { basal, los terminales trilobados. } \\
\text { Pedicelos florales } 0,6-1 \mathrm{~mm} \text { de } \\
\text { largo. Flores (amarillas) y frutos } \\
\text { dispuestos en racimos de ca. } 10 \\
\text { silicuas, éstas de } 0,5-0,8 \mathrm{~cm} \text { de } \\
\text { largo por } 0,1-0,2 \mathrm{~cm} \text { de ancho, } \\
\text { generalmente agrupadas en el } \\
\text { centro de la roseta. }\end{array}$ & $\begin{array}{l}\text { A lo largo de la Cordillera de Los } \\
\text { Andes, desde Colombia a través } \\
\text { de Ecuador, Perú y Bolivia, y } \\
\text { hasta el altiplano en el noroeste } \\
\text { de Argentina, entre los } 2.500 \\
\text { y los } 4.200 \text { m snm. También } \\
\text { presente en el Archipiélago de } \\
\text { Las Galápagos (Santiago). En } \\
\text { Chile crece al noroeste de la } \\
\text { Patagonía, entre los } 700 \text { y los } \\
1.000 \text { m snm. Probablemente } \\
\text { también esté presente en la } \\
\text { Patagonía argentina nororiental } \\
\text { y en Chile al oeste de la Región } \\
\text { de Antofagasta. }\end{array}$ & $\begin{array}{l}\text { Chile, al este de } \\
\text { Nirehuao camino } \\
\text { a Coyhaique Alto, } \\
\text { charco temporal, } \\
\text { 21-IV-2011, M. } \\
\text { Álvarez } 882 \text { (FB). }\end{array}$ \\
\hline
\end{tabular}

oligophylla) y probablemente son caracterizadas por sustratos más halófilos o eutróficos. Ambas alianzas han sido descritas para la Patagonia argentina suroriental (Deil et al. 2011). Sin embargo la clasificación de estas comunidades requiere de estudios más detallados en base a censos vegetales y descripciones del ambiente.
A la falta de conocimiento sobre la ecología de este tipo de hábitats se suma la ausencia de medidas de protección de lagunas temporales, las cuales están siendo afectadas por su uso desmesurado como bebederos para el ganado vacuno y ovino que pastorea en las estepas y praderas aledañas. También se observaron en terreno remociones 
del sustrato hechas por jabalies. Por ende, se hace urgente realizar estudios más detallados para determinar el estado de riesgo de estas comunidades, las cuales no sólo sirven de refugio a especies vegetales endémicas con un alto potencial de riesgo de conservación, sino que también cumplen funciones ecológicas al ofrecer hábitat para la ornitofauna local. Un análisis más detallado de los riesgos para la flora acuática de la Región de Aisén lo presentan San Martín et al. (2011).

\section{AGRADECIMIENTOS}

A la CONAF, Chile por el financiamiento parcial del Proyecto "Actualización Catastro de Recursos Vegetacionales, Región de Aisén”. Al Prof. Ulrich Deil (Freiburg, Alemania) por su inspiración $e$ incentivo al estudio de lagunas temporales en Sudamérica. Visitas a terreno han sido financiadas por la A.F.W. Schimper-Stiftung (Alemania).

\section{LITERATURA CITADA}

Álvarez, M. (2008). Diasporenbank und Ökologie der Vegetation temporärer Gewässer im Cholchol-Gebiet (9. Region, Chile). J. Cramer, Berlin. Dissertationes Botanicae 407: 1-87.

Álvarez, M. J. San Martín \& U. Deil (2012). Nanism and ephemerism as reasons for a hidden abundance in vernal pool plants: the example of Lepuropetalon spathulatum in Chile. Feddes Repertorium, 123: 55-66.

Arroyo, M., T. K., C. Marticorena, O. Matthei, M. Muñoz \& P. Pliscoff (2002). Analysis of the contribution and efficiency of the Santuario de la Naturaleza Yerba Loca, $33^{\circ} \mathrm{S}$ in protecting the regional vascular plant flora (Metropolitan and Fifth regions of Chile). Revista Chilena de Historia Natural 75: 767-792.

Arroyo, M. T. K., C. Marticorena \& M. Muñoz (1990). A checklist of the native annual flora of continental Chile. Gayana Botánica 47: 119-135.

Bliss, S. A., P. H. Zedler, J. E. Keeley \& M. T. K. Arroyo (1998). A floristic survey of the temporary wetlands in the Mediterranean-climate region of Chile. In: A. J. McComb \& J. A. Davis (eds.), Wetlands for the future (pp.
219-228). Adelaide: Gleneagles Press.

Clausen, J. C., I. M. Ortega, C. M. Glaude, R. A. Relyea, G. Garay \& O. Guineo (2006). Classification of wetlands in a Patagonian National Park, Chile. Wetlands 26: 217-229.

Collantes, M. B. \& A. M. Faggi (1999). Los Humedales del sur de Sudamérica. In: A. I. Malvárez (ed.), Tópicos sobre humedales subtropicales y templados de Sudamérica (pp. 14-24) Montevideo: UNESCO, MAB.

Cooper, D.J., E. C. Wolf, C. W. Colson, W. A. Vering, A. Granda \& M. Meyer (2010). Alpine peatlands of the Andes, Cajamarca, Peru. Arctic, Antarctic, and Alpine Research 42: 19-33.

Deil, U. (2005). A review on habitats, plant traits and vegetation of ephemeral wetlands - a global perspective. Phytocoenologia 35: 533-705.

Deil, U. \& M. Álvarez (2012). Database of ephemeral wetland vegetation in extra- and oro-tropical South America. Biodiversity and Ecology 4: 441.

Deil, U., M. Álvarez \& I. Paulini (2007). Native and non-native species in annual grassland vegetation in Mediterranean Chile. Phytocoenologia 37: 769-784.

Deil, U., M. Álvarez, E-M. Bauer \& C. Ramírez (2011). The vegetation of seasonal wetlands in extratropical and orotropical South America. Phytocoenologia 41: 1-34.

Faggi, A.M. (1985). Las comunidades vegetales de Río Gallegos, Santa Cruz. En: Boelcke, O., D.M. Moore \& F.A. Roig (eds.) Transecta botánica de la Patagonia Austral. (pp. 592-633). Buenos Aires: Consejo Nacional de Investigaciones Científicas y Técnicas (Argentina), Instituto de la Patagonia (Chile), Royal Society (Gran Bretaña).

Fontúrbel, F., E. Richard \& G. García-Crispieri (2006). Diversidad de flora fanerógama y aspectos ecobiológicos de la unidad vegetal de Aygachi-Cumana-Bahía Cohana, Lago Titikaka (La Paz, Bolivia). Ciencia Abierta Internacional 30: 1-19.

Gobierno Regional de Aysén \& Gesellschaftfür Technische Zuzammenarbeit (2005). Atlas Región de Aysén. Santiago: LOM.

Horn, N. (2000). Revision der Gattungen Plagiobothrys und Pectocarya in Chile und den angrenzenden Gebieten. München: Thesis 
Phd, Ludwig-Maximilians-Universität.

Jaramillo-Díaz, P., A. Guézou, A. Mauchamp \& A. Tye (2011). Charles Darwin Foundation checklist of Galapagos flowering plants. Galapagos: Charles Darwin Foundation.

León, R. J., C. D. Bran, M. B. Collantes, J. M. Paruelo \& A. Soriano (1998). Grandes unidades de vegetación de la Patagonia extra andina. Ecología Austral 8: 125-144.

Luebert, F. \& P. Pliscoff (2006). Sinopsis bioclimática $y$ vegetacional de Chile. Santiago: Editorial Universitaria.

Marticorena, C. \& M. Quezada (1985). Catálogo de la flora vascular de Chile. Gayana Botánica 42: 1-147.

Martínez-Carretero, E. (2004). Los turbales patagónicos. En: Blanco, D.E. \& V.M. de la Balze (eds.), Los turbales de la Patagonia. Buenos Aires: Wetlands International.

Méndez, E. \& A. J. Ambrosetti (1985). Las comunidades vegetales de Río Turbio, El Turbio, Santa Cruz. En: Boelcke, O., D.M. Moore, \& F.A. Roig (eds.) Transecta botánica de la Patagonia Austral. Buenos Aires: Consejo Nacional de Investigaciones Científicas y Técnicas (Argentina), Instituto de la Patagonia (Chile), Royal Society (Gran Bretaña).

Ortuño, T., S. G., Beck \& L. Sarmiento (2006). Dinámica sucesional de la vegetación en un sistema agrícola con descanso largo en el altiplano central boliviano. Ecología en Bolivia 41: 40-70.

Parra, C. \& J. L. Fernández Alonso (2002). Adiciones a la flora de Colombia: novedades taxonómicas, corológicas y sinopsis de la tribu Arabideae (Brassicaceae). Caldasia 24: 323-341.

Roig, F. A. (1998). La Vegetación de la Patagonia. Mendoza: Consejo Nacional de Investigaciones Científicas y Técnicas.

Roig, F. A., J. Anchorena, O. Dollenz, O. Faggi, A.
M. \& E. Méndez (1985a). Las comunidades vegetales de la transecta botánica de la $\mathrm{Pa}$ tagonia Austral. Primera parte: la vegetación de área continental. En: Boelcke, O., D.M. Moore, \& F.A. Roig (eds.), Transecta botánica de la Patagonia Austral. Buenos Aires: Consejo Nacional de Investigaciones Científicas y Técnicas (Argentina), Instituto de la Patagonia (Chile), Royal Society (Gran Bretaña).

Roig, F.A., O. Dollenz \& E. Méndez (1985b). Las comunidades vegetales de la transecta botánica de la Patagonia Austral. Segunda parte: la vegetación en los canales. En: Boelcke, O., D.M. Moore, \& F.A. Roig (eds.) Transecta botánica de la Patagonia Austral. Buenos Aires: Consejo Nacional de Investigaciones Científicas y Técnicas (Argentina), Instituto de la Patagonía (Chile), Royal Society (Gran Bretaña).

Ruthsatz, B. (1977). Pflanzengesellschaften und ihre Lebensbedingungen in den Andinen Halbwüsten Nordwest-Argentiniens. Dissertationes Botanicae 39: 1-168.

San Martín, C. \& M. Alvarez (2009). Floristic composition of anthropogenic seasonal wetlands in the coastal mountain range of Cautín, Chile. Agro Sur 37: 9-25.

San Martín, C., Y. Pérez, D. Montenegro \& M. Alvarez (2011). Diversidad, hábito y hábitat de macrófitos acuáticos en la Patagonia occidental (Región de Aisén, Chile). Anales Instituto de la Patagonia 39(1): 23-41.

Servicio Nacional de Geología y Minería (2003). Mapa Geológico de Chile. Santiago: Servicio Nacional de Geología y Minería.

Zuloaga, F. O., O. Morrone \& M. J. Belgrano (2008). Catálogo de las plantas vasculares del cono sur. Missouri: Missouri Botanical Gardens. 3 vols. 